\title{
DOG1: a novel marker of salivary acinar and intercalated duct differentiation
}

\author{
Jacinthe Chênevert ${ }^{1}$, Umamaheswar Duvvuri ${ }^{2}$, Simion Chiosea ${ }^{1}$, Sanja Dacic ${ }^{1}$, \\ Kathleen Cieply ${ }^{1}$, Jean Kim², Daniel Shiwarski ${ }^{2}$ and Raja R Seethala ${ }^{1,2}$ \\ ${ }^{1}$ Department of Pathology, University of Pittsburgh Medical Center, Pittsburgh, PA, USA and \\ ${ }^{2}$ Department of Otolaryngology, University of Pittsburgh Medical Center, Pittsburgh, PA, USA
}

\begin{abstract}
Anoctamin-1 (ANO1) (DOG1, TMEM16a) is a calcium-activated chloride channel initially described in gastrointestinal stromal tumors, but now known to be expressed in a variety of normal and tumor tissues including salivary tissue in murine models. We herein perform a comprehensive survey of DOG1 expression in 156 cases containing nonneoplastic human salivary tissues and tumors. ANO1 mRNA levels were significantly higher (8-fold increase, $P<0.0001)$ in normal parotid tissue $(n=6)$ as compared with squamous mucosa $(n=15)$. By immunohistochemistry, DOG1 showed a diffuse moderate $(2+)$ apical membranous staining pattern in normal serous acini, $1+$ apical membranous pattern in mucous acini, and variable 1-2 + apical staining of distal intercalated ducts. Myoepithelial cells, striated and excretory ducts were invariably negative. All acinic cell carcinomas $(n=28)$ were DOG1 positive demonstrating a complex mixture of intense $(3+)$ apical membranous, cytoplasmic and complete membranous staining. Most ductal tumor types were negative or only showed a subset of positive cases. Within the biphasic tumor category, adenoid cystic carcinomas (18/24 cases) and epithelial-myoepithelial carcinomas (8/15 cases) were frequently positive, often showing a distinctive combined apical ductal and membranous/cytoplasmic myoepithelial staining profile. Thus, DOG1 staining is a marker of salivary acinar and to a lesser extent intercalated duct differentiation. Strong staining can be used to support the diagnosis of acinic cell carcinoma. DOG1 may also be a marker of a 'transformed' myoepithelial phenotype in a subset of biphasic salivary gland malignancies.
\end{abstract} Modern Pathology (2012) 25, 919-929; doi:10.1038/modpathol.2012.57; published online 30 March 2012

Keywords: acinic cell carcinoma; DOG1; salivary gland

The gene now known as anoctamin-1 (ANO1, also known as FLJ10261, TMEM16A, discovered on GIST1, or DOG1) was initially noted by gene expression profiling to be differentially expressed in gastrointestinal tumors (GIST) ${ }^{1}$ when compared with a variety of other mesenchymal tumors. Since then, the expression of the ANO1, more commonly known as DOG1, by immunohistochemistry has been validated on large series of GIST, but has also been noted in other tumor types such as esophageal and head and neck squamous cell carcinoma. ${ }^{2-4}$ Its main function as a calcium-activated chloride channel was uncovered in 2008. ${ }^{5-7}$ Structurally, DOG1 protein is predicted to contain eight transmembrane segments, hence the official designation ANO1. ${ }^{8}$

To date, the main clinical application of DOG1 immunostaining has been as a marker of GIST, in

Correspondence: Dr RR Seethala, MD, Department of Pathology, University of Pittsburgh Medical Center, Scaife Hall A616.3, 200 Lothrop Street, Pittsburgh, PA 15213, USA.

E-mail: seethalarr@upmc.edu

Received 24 August 2011; revised 15 February 2012; accepted 16 February 2012; published online 30 March 2012 surgical and even cytologic specimens. ${ }^{2,3,9}$ However, the discovery of its calcium-activated chloride channel properties has suggested a potential role in secretory cell types such as those of the salivary gland, and thus perhaps tumors derived from these cell types. Interestingly, studies in murine models have demonstrated that DOG1 is not only present but also required for normal salivary gland secretory activity. ${ }^{10-12}$ However, DOG1 expression has not been well studied in human salivary tissues. Only a few normal salivary glands and salivary tumors have been stained, mainly in the context of broad immunohistochemical surveys in reference to its performance as a marker of GIST. ${ }^{2}$

The aim of this study is to characterize DOG1 expression patterns in normal human salivary tissues and a broad variety of salivary gland tumors.

\section{Materials and methods}

This study was approved by our institutional review board (IRB\#PRO07050360). 


\section{qRT-PCR for ANO1 mRNA}

In all, 6 normal parotid and 15 non-cancerous squamous mucosal snap-frozen tissue specimens (stored at $-80^{\circ} \mathrm{C}$ ) were retrieved from the institutional tissue biorepository for comparative analysis. ANO1 mRNA levels were evaluated by quantitative reverse transcription PCR (qRT-PCR) Taqman primers and probes were designed with the PRIMER EXPRESS V.2.0.0 program (Applied Biosystems, Foster City, CA). The reverse transcriptions were carried out as previously described. ${ }^{13}$ Quantitative PCR (qPCR) was performed on the cDNA using the ABI 7700 Sequence Detection Instrument (Applied Biosystems) and analyzed using the relative quantification method. qPCR was performed for $A N O 1$ and $G A P D H$ was used as an endogenous control. For the qPCR, the final concentrations of the reaction components were as follows: $1 \times$ Taqman Universal PCR Master Mix (Applied Biosystems), $500 \mathrm{nM}$ Forward (GAGCCAAAGACATCGGAATCTG) and Reverse (TGAAGGAGATCACGAAGGCAT) primers, 200 nM Florescent Probe (FAM-CTCAGAGGCA TTGGGAAGCTTGCTGT-TAMARA), and DNAsefree $\mathrm{H}_{2} \mathrm{O}$ to a final volume of $25 \mu \mathrm{l}$. The primer and probe concentration have been described previously. ${ }^{4}$ The thermocycler conditions were $95^{\circ} \mathrm{C}$ Taq activation for $12 \mathrm{~min}$ and 40 cycles of $95^{\circ} \mathrm{C}$ denaturation for $15 \mathrm{~s}$ followed by $60^{\circ} \mathrm{C}$ anneal/ extend for $60 \mathrm{~s}$. The consistency of amplification was judged by inspection of the melting curves. The DDCt method was used to determine relative expression. A standard curve was also performed to evaluate the efficiency of the qPCR experiment. The differences in relative expression were determined for each sample, run in triplicate, using a Student's $t$-test via GraphPad Prism software.

\section{Case Selection for DOG1 Immunohistochemical Validation}

A total of 156 formalin-fixed paraffin-embedded cases were retrieved from the University of Pittsburgh Medical Center pathology archives. The neoplastic cases $(n=150)$ retrieved were as follows: 28 acinic cell carcinomas, 24 adenoid cystic carcinomas, 16 polymorphous low-grade adenocarcinomas, 15 epithelial - myoepithelial carcinomas, 14 pleomorphic adenomas, 12 myoepithelial tumors (4 myoepitheliomas/8 myoepithelial carcinomas), 10 oncocytic tumors (8 oncocytomas/2 oncocytic carcinomas), 9 salivary duct carcinomas, 8 mucoepidermoid carcinomas, 6 mammary analog secretory carcinomas, and 8 others (2 Warthin tumors, 2 low-grade salivary duct carcinomas/low-grade cribriform cystadenocarcinomas, 1 adenosquamous carcinoma, and 1 sebaceous lymphadenoma). Six additional normal or non-neoplastic cases were also retrieved: three submandibular glands with chronic sialadenitis, one parotid gland with sialadenosis, one nasal septum biopsy with normal mucoserous glands, and one containing normal serous (Von Ebner) and mucinous glands of the base of tongue.

\section{DOG1 Immunohistochemistry}

Formalin-fixed paraffin-embedded sections were mounted, and serially sectioned at $4 \mu \mathrm{m}$ intervals. Sections were deparaffinized and subjected to heatinduced epitope retrieval in citrate buffer. Immunohistochemical staining was performed using DOG1 (Clone 1.1; Zeta Co, Sierra Madre, CA; dilution 1:50). Labeling was performed using the I-view $2^{\prime}$-diaminobenzamide (DAB) detection kit (Ventana systems, Tucson, AZ) as the brown chromogen substrate.

Staining parameters evaluated included: cell type, $\%$ of cells stained, intensity (scale: 0-3), and subcellular localization: membranous-including location and extent (ie, apical-luminal, basolateral, and complete), cytoplasmic, and other. These parameters were collected for the tumor and also for adjacent normal tissue if it was present in the same section. Case was considered as 'negative' or as having 'negligible staining' if $<2 \%$ of the tumor expressed DOG1, as 'focal' if between 2 and $50 \%$, and as 'diffuse' if $>50 \%$ had staining. As for the intensity of staining, the apical staining of normal parotid serous acini was used as $2+$; a more intense staining was graded as $3+$ and weaker staining as $1+$.

\section{ETV6-NTRK3 Fluorescence In-Situ Hybridization}

Six cases morphologically compatible with the diagnosis of mammary analog secretory carcinomas were confirmed for an ETV6-NTRK3 translocation as described previously ${ }^{14-16}$ using ETV6 break apart probe set.

Briefly, formalin-fixed paraffin-embedded sections were mounted, and serially sectioned at $5 \mu \mathrm{m}$ intervals and incubated with the Vysis LSI ETV6 (TEL) (12p13) Dual Color, Break Apart Rearrangement Probe overnight at $37^{\circ} \mathrm{C}$ in a humidified chamber and counterstained with DAPI (Vysis). Analysis was performed using an Applied Imaging Workstation equipped with Chroma Technology filters containing band excitors for SpectrumOrange, FITC, and DAPI. Only individual and well-delineated cells were scored, overlapping cells were excluded and $\sim 60$ cells were analyzed in the targeted region. A case was considered positive if a split green and orange signal was identified in $>20 \%$ of the nuclei.

\section{Results}

ANO1 mRNA Expression in Salivary Frozen Tissues

ANO1 mRNA levels were significantly higher (8-fold increase, $P<0.0001$ ) in normal parotid tissue as compared with normal squamous epithelium (Figures 1a and b). 
DOG1 Immunohistochemical Staining Profile in Normal, Non-Neoplastic Salivary Gland

Normal salivary gland tissue was present in 109 cases (103 peritumoral normal cases and 6 nonneoplastic cases). Staining was greatest in salivary acini. Regardless of salivary subsite, normal serous acini uniformly demonstrated a sharp, crisp, moderately $(2+)$ intense membranous staining in an apical-luminal pattern with occasional basolateral staining (Figures 2a and c). Mucous acini showed a similarly well-delineated apical pattern of membranous staining, but with less intensity $(1+$, Figures $2 \mathrm{~b}$ and $\mathrm{c}$ ). Intercalated ducts were variably positive in an apical-luminal membranous pattern. Staining of intercalated ducts was more frequent in the more distal portions toward the acini and ranged from $1+$ to $2+$ (Figure 2a, single arrow). In all cases, striated ducts (Figure 2a, letter ' $x$ ') and more proximal larger excretory ducts were negative for DOG1. No normal constituent showed cytoplasmic staining, or complete membranous staining. Three cases of sialadenitis and one case of sialadenosis (Figure 2d) showed a similar staining pattern in each of the ductoacinar components.

\section{DOG1 Expression in Acinar and Ductal Tumors}

Immunostaining of all salivary tumors is summarized in Table 1. All 28 acinic cell carcinomas showed DOG1 staining, and 93\% (26/28) tumors showed diffuse $(>50 \%)$ staining. Overall, staining was more intense and more complex than in normal acini with $25 / 28(89 \%)$ of acinic cell carcinomas showing $3+$ apical/luminal membranous staining as well as scattered foci of complete membranous and even cytoplasmic staining (Figures 3a and b). Acinar cell types stained most intensely, while intercalated ductal, non-specific ductal, and vacuolated cells were more variable and somewhat weaker in DOG1 staining intensity, and in some areas negative. When present, the membranous and cytoplasmic staining was weaker than the apical pattern, with an intensity of $1+$ or $2+$. Two acinic cell carcinomas $(7 \% ; 2 / 28)$ showed only focal DOG1 staining. However, these cases had a prominence of vacuolated and nonspecific ductal cells with a paucity of acinar cells (Figures 3c and d).

Six cases of mammary analog secretory carcinoma (Figure 4a), a new entity historically categorized as acinic cell carcinomas, were confirmed by fluorescence in-situ hybridization (FISH) (Figure 4b) and subsequently tested for DOG1. Staining was observed in $2 / 6$ (33\%) of cases. One case showed fairly diffuse $(80 \%)$ staining reminiscent of acinic cell carcinomas with $2+$ apical membranous and $1+$ cytoplasmic staining pattern. A second case was focally positive $(40 \%)$ with faint $(1+)$ apical and complete membranous staining. The remainder of mammary analog secretory carcinomas were negative (Figure 4c).

Of the other phenotypically ductal tumors, polymorphous low-grade adenocarcinoma most frequently demonstrated DOG1 immunopositivity, present in 31\% (5/16) cases; however, staining was very focal in all but one case. Staining was not nearly as strong as in acinic cell carcinomas and was more reminiscent of normal intercalated ducts with a 1-2+ apical-luminal pattern of staining. The single diffusely positive case also showed areas of more complete membranous staining (Figures $5 \mathrm{a}$ and b). There were no striking differences in histologic growth pattern (ie, proportion of papillary components) between the positive and negative polymorphous low-grade adenocarcinoma. All nine salivary duct carcinomas (Figures 5c and d), eight oncocytomas, two oncocytic carcinomas, and two low-grade salivary duct carcinomas (a.k.a. low-grade cribriform cystadenocarcinomas) tested were negative for DOG1.

\section{DOG1 Expression in Biphasic Salivary Gland Tumors}

Twenty-four adenoid cystic carcinomas were evaluated: 4 tubular pattern predominant, 14 cribriform
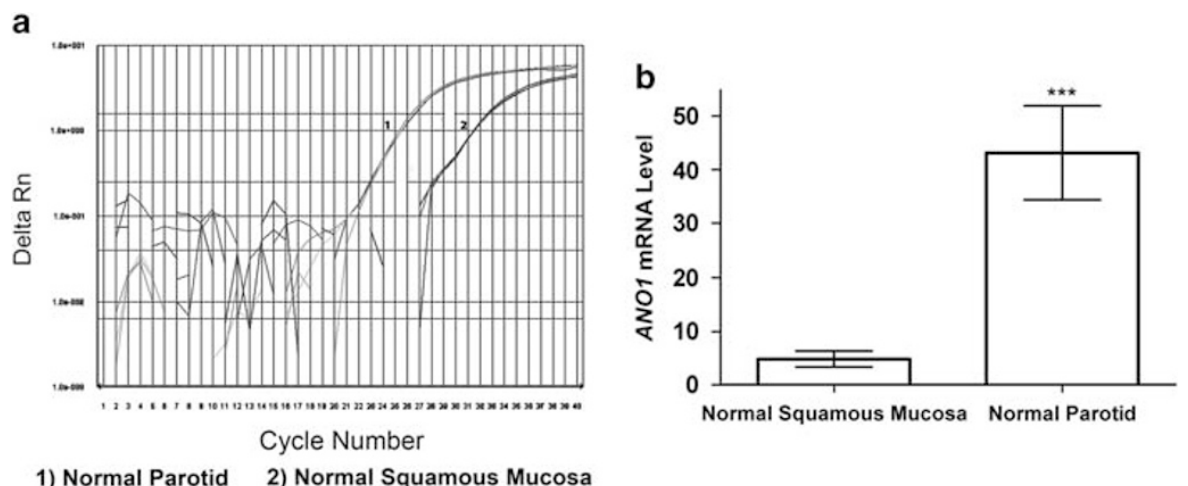

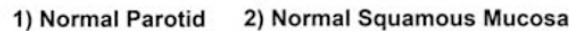

Figure 1 qRT-PCR amplification of ANO1 in normal parotid tissue and normal squamous mucosa. (a) Parotid tissue samples (curve aggregate 1) show a $\mathrm{C}_{t}$ that is earlier that of the normal squamous mucosa (curve aggregate 2). (b) Relative expression levels of $A N O 1$ mRNA showing an 8-fold higher level of expression in parotid tissue as compared with normal squamous mucosa $\left({ }^{* * *} P<0.0001\right)$. Box edge-mean value, whiskers standard error of mean. 

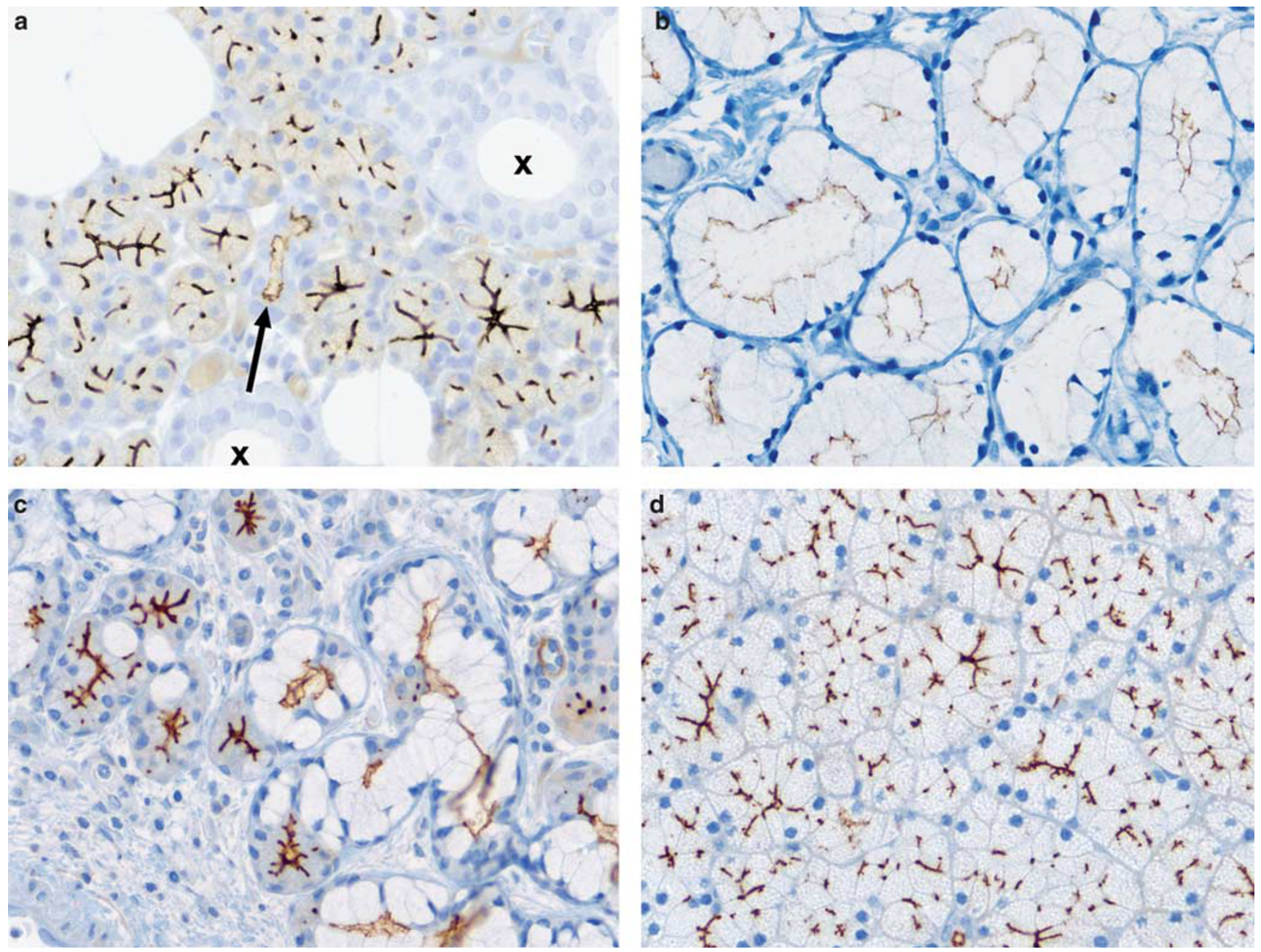

Figure 2 DOG1 staining pattern in normal salivary gland tissues (all $\times 400)$. (a) Serous acini of parotid with moderate $(2+)$ apicalluminal membranous staining and weaker $(1+)$ intercalated duct (arrow) staining. Striated ducts are negative (x mark). (b) Mucous acini in a minor salivary gland with $1+$ apical staining. (c) Sinonasal mucoserous glands with stronger expression in serous acini compared with mucous acini. (d) Sialadenosis of parotid showing almost equivalent DOG1 intensity as compared with normal parotid.

pattern predominant, 3 solid pattern predominant, and 3 adenoid cystic carcinoma with high-grade transformation. Overall, 75\% (18/24) adenoid cystic carcinoma stained for DOG1, and 38\% (9/24) showing diffuse staining. When present, staining was frequently noted in both ductal and myoepithelial cells (Figures 6a and b). However, the ductal components showed an apical-luminal staining pattern similar to normal intercalated ducts $(1-2+$ intensity), while the myoepithelial cells showed diffuse cytoplasmic staining $(2+$ intensity). The tubular and cribriform predominant adenoid cystic carcinoma was more frequently DOG1 positive (89\%, 16/18 cases) as compared with solid adenoid cystic carcinoma and adenoid cystic carcinoma with high-grade transformation $(33 \%, 2 / 6$ cases). One case of adenoid cystic carcinoma with high-grade transformation did show some DOG1 positivity, but only in the adjacent conventional adenoid cystic carcinoma component.
In all, 15 epithelial-myoepithelial carcinomas were evaluated: 14 cases had classic clear cell morphology, while 1 case was an oncocytic-sebaceous variant. Three of the classic epithelial-myoepithelial carcinomas arose in a pleomorphic adenoma, and one was associated with intercalated duct hyperplasia (see also below). Epithelial-myoepithelial carcinoma frequently showed DOG1 positivity with $53 \%(8 / 15)$ showing positivity, and $47 \%(7 / 15)$ showing diffuse positivity. Staining was similar to that seen in adenoid cystic carcinoma (Figures 6c and d). When positive, the ductal components showed 1-2+ apical-luminal staining, while the myoepithelial components showed 1-2+ complete membranous or cytoplasmic staining. There were no morphologic differences between positive and negative cases. The oncocytic-sebaceous epithelial-myoepithelial carcinoma showed staining in only the myoepithelial component with $2+$ complete membranous positivity. 
Table 1 DOG1 staining in salivary gland neoplasms

\begin{tabular}{lcccc}
\hline Diagnosis & $\begin{array}{c}\text { Negative } \\
\text { or } \leq 2 \%\end{array}$ & $\begin{array}{c}\text { Focal } \\
\text { positivity } \\
(3-50 \%)\end{array}$ & $\begin{array}{c}\text { Diffuse } \\
\text { positivity } \\
(>50 \%)\end{array}$ & Total \\
\hline $\begin{array}{l}\text { Acinic cell carcinoma } \\
\begin{array}{l}\text { Mammary analog } \\
\text { secretory carcinoma }\end{array}\end{array}$ & 4 & 2 & 26 & 28 \\
$\begin{array}{l}\text { Polymorphous low-grade } \\
\text { adenocarcinoma }\end{array}$ & 11 & 4 & 1 & 16 \\
$\begin{array}{l}\text { Salivary duct carcinoma } \\
\text { Adenoid cystic carcinoma }\end{array}$ & 9 & - & - & 9 \\
$\begin{array}{l}\text { Epithelial-myoepithelial } \\
\text { carcinoma }\end{array}$ & 7 & 8 & 9 & 24 \\
$\begin{array}{l}\text { Pleomorphic adenoma } \\
\begin{array}{l}\text { Mucoepidermoid } \\
\text { carcinoma }\end{array}\end{array}$ & 13 & 1 & 7 & 15 \\
$\begin{array}{l}\text { Myoepithelioma/ } \\
\text { myoepithelial carcinoma }\end{array}$ & $4 / 7$ & $-/ 1$ & - & 14 \\
$\begin{array}{l}\text { Oncocytoma/oncocytic } \\
\text { carcinoma }\end{array}$ & $8 / 2$ & - & - & 10 \\
\begin{tabular}{l} 
Other \\
\hline
\end{tabular} & $6^{\mathrm{b}}$ & $1^{\mathrm{c}}$ & $1^{\mathrm{d}}$ & 8 \\
\hline
\end{tabular}

${ }^{\mathrm{a}}$ Includes three high-grade transformations of adenoid cystic carcinomas.

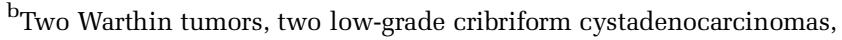
one adenosquamous carcinoma, and one sebaceous lymphadenoma.

${ }^{\mathrm{C}}$ One basal cell adenocarcinoma.

${ }^{\mathrm{d}}$ One intercalated duct adenoma.

Of the other biphasic salivary gland neoplasms, the one basal cell adenocarcinoma tested was focally positive ( $40 \%$ of tumor) in the basal cell component in a $2+$ cytoplasmic and membranous pattern; predominant growth patterns were solid and membranous. Only 1/13 (8\%) pleomorphic adenoma showed even focal (5\%) DOG1 staining, with a $1+$ apical staining pattern in the ductal component only.

\section{Other Salivary Gland Neoplasms and Precursor Lesions}

In all, $38 \%(3 / 8)$ of the mucoepidermoid carcinomas (1 low grade and 2 intermediate grade) studied showed focal $(3-20 \%)$ apical $1+$ staining in the mucous cell components. Only 14\% (1/7) myoepithelial carcinomas with spindle cell predominant histology showed DOG1 staining, but only focally with $1+$ cytoplasmic staining in $20 \%$ of the tumor. All four myoepitheliomas were negative. All Warthin tumors and other tested tumors were negative.

Interestingly, four cases showed putative precursor lesions (three intercalated duct hyperplasias and one intercalated duct adenoma). The intercalated duct hyperplasias were seen adjacent to or in association with tumors mentioned above (one mammary analog secretory carcinoma, one epithelial-myoepithelial carcinoma, and one cystadenoma). The intercalated duct adenoma on the other hand was seen in isolation. All precursor lesions were positive for DOG1 and 3/4 (75\%) showed diffuse positivity. All cases showed apical staining ranging from 1 to $3+$. The intercalated duct adenoma showed $3+$ apical staining and $1+$ cytoplasmic staining of myoepithelial cells as well (Figures 6e and f).

\section{Discussion}

The discovery of the function of ANO1 (DOG1) as a calcium-dependent chloride channel suggests potential roles for this protein beyond that of a marker of GIST. Indeed, recently, DOG1 has been characterized in pancreatic centroacinar cells and a subset of islet cells suggesting a potential exocrine/endocrine secretory role. ${ }^{17,18}$ With respect to salivary gland, DOG1 has been shown to be expressed and perhaps even required for normal salivary secretion in murine models. ${ }^{10-12}$ Thus perhaps not surprisingly, we were able to confirm high levels of ANO1 mRNA and DOG1 protein by immunohistochemistry in human salivary tissues as well in this study, which represents the first comprehensive survey of non-neoplastic salivary tissues and salivary gland neoplasms.

The high level of DOG1 expression in salivary tissues was found to be localized immunohistochemically mainly to salivary serous acini, which invariably showed an apical pattern of staining of moderate intensity. Mucous acini also consistently showed a similar pattern but with lesser intensity. This staining diminished at the level of the intercalated ducts and was completely absent more proximally. This pattern of staining is in keeping with the function of DOG1 as a transmembrane anion channel with a secretory role. Of note, no cytoplasmic staining was noted in normal salivary gland. This is in contrast to other cell types in which DOG1 is constitutively expressed such as interstitial cells of Cajal where cytosolic localization of DOG1 is typical. ${ }^{19,20}$ Potential reasons for this may include a differential distribution of isoforms, and differences in post-translational modification and processing in the endoplasmic reticulum and Golgi apparatus, and potentially even the presence in some cell types of lipid microvacuoles with DOG1 that are exocytosed in response to increases in cytosolic calcium. ${ }^{11,19}$

As expected, acinic cell carcinomas are the tumor types with the strongest expression of DOG1. In contrast to normal acini, staining was noted to be more intense and more complex often with cytoplasmic staining. This study was not intended to address the potential reasons for this apparent increase in DOG1 staining. The high expression in acinic cell carcinomas may simply represents an 'exaggerated acinar' phenotype rather than a specific event such as gene amplification as seen in some tumors with DOG1 overexpression. For instance in squamous cell carcinoma of the head and neck, the chromosome 11q13 region in which the ANO1 gene resides is frequently amplified suggesting a potential mechanism for DOG1 overexpression in a subset of these tumors., ${ }^{41}$ However, this region has not been described to be amplified in salivary acinic cell carcinomas. 

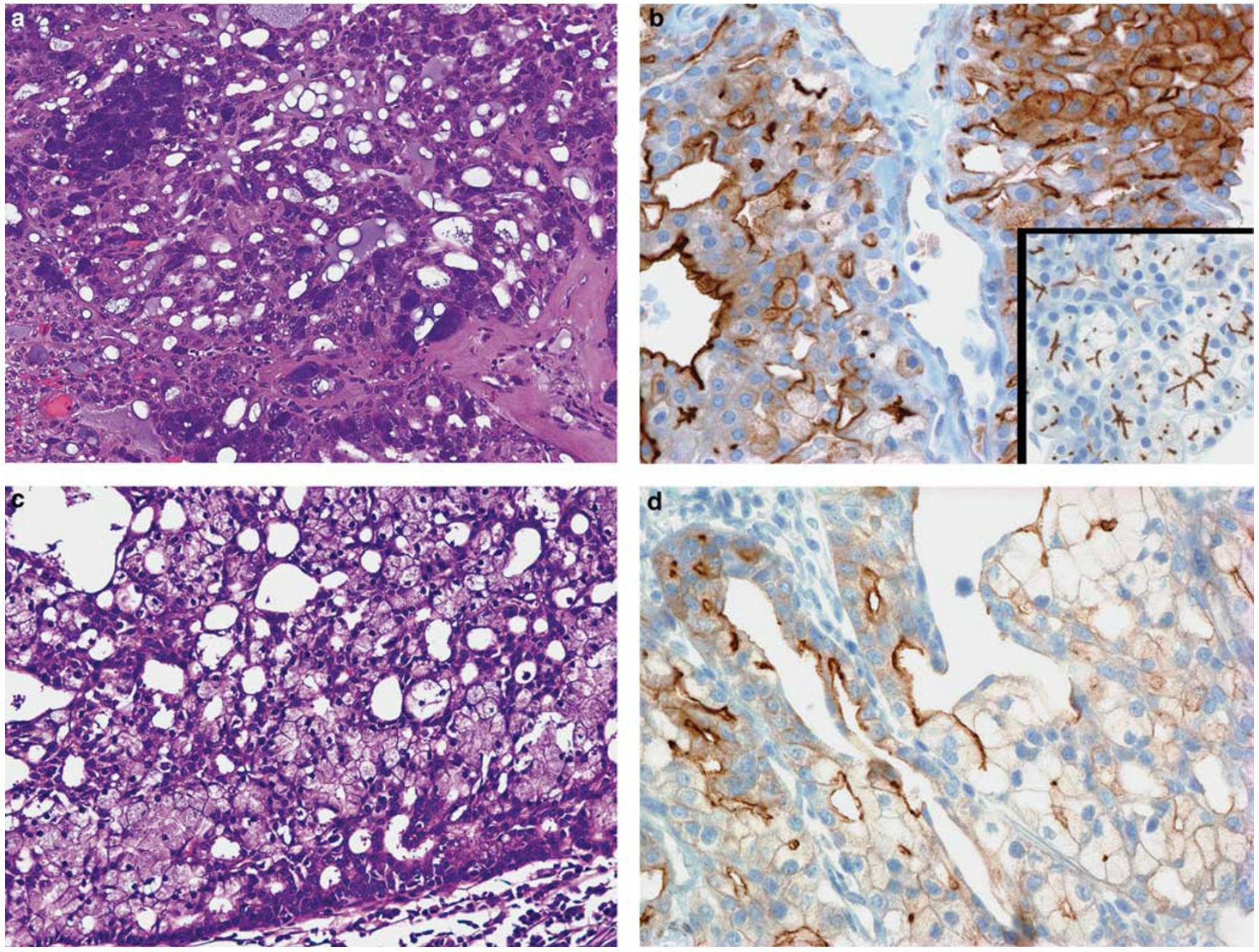

Figure 3 DOG1 in acinic cell carcinoma. (a) Acinic cell carcinoma, with abundant acinar cell components (H\&E, $\times 200)$. (b) DOG1 staining showing intense $(3+)$ apical membranous staining around lumina as well as complete membranous and variable cytoplasmic staining $(1-2+)(\times 400)$. Inset: adjacent non-neoplastic parotid for comparison $(\times 400)$. (c) Acinic cell carcinoma with predominant vacuolated and non-specific ductal cells and only scattered acinar cells (H\&E, $\times 200)$. (d) DOG1 staining showing only focal $2+$ apical positivity (left) and faintly positive $1+$ to negative complete membranous staining in the vacuolated areas (right) $(\times 400)$.

Regardless of mechanism, our findings would indicate that this consistently strong DOG1 staining can be utilized diagnostically to support the diagnosis of acinic cell carcinomas. Typically, acinic cell carcinomas show readily recognizable serous acinar differentiation on a routine hematoxylin and eosinstained slide. When this cell type is less prominent, traditionally, the histochemical stain using period acid-Schiff reaction after diastase digestion can be used to highlight zymogen granules. However occasionally, even these may be scarce, and in some cases, non-acinic cell carcinomas may show small globules of intracytoplasmic mucin or even hemosiderin that may be periodic acid-Schiff positive and diastase resistant. In such situations, it is appropriate to exclude these 'zymogen granule mimics' by performing a mucicarmine, and in some instances, an iron stain. Anti-amylase immunostains may be useful for the diagnosis of acinic cell carcinomas if positive $;^{22,23}$ however, the sensitivity of this marker for acinar differentiation is very low. Thus, DOG1 staining offers a sensitive and robust marker to support the diagnosis of acinic cell carcinomas.

The differential diagnosis for acinic cell carcinomas can potentially be quite broad depending on histologic pattern and cell type predominance; however, in most cases, it can be resolved to tumors that are phenotypically ductal. Thus when considering this subgroup of tumors, DOG1 also appears to show a good deal of specificity, with only occasional ductal tumors showing immunopositivity that approaches the extent seen in acinic cell carcinomas. For instance, all salivary duct carcinomas, oncocytomas, oncocytic carcinomas, and low-grade salivary duct carcinomas tested were negative. Only a subset of polymorphous low-grade adenocarcinoma was DOG1 positive, with only one diffusely positive tumor. Interestingly, the focality and heterogeneity of reactivity in polymorphous low-grade adenocarcinoma is reminiscent of the normal staining profile 

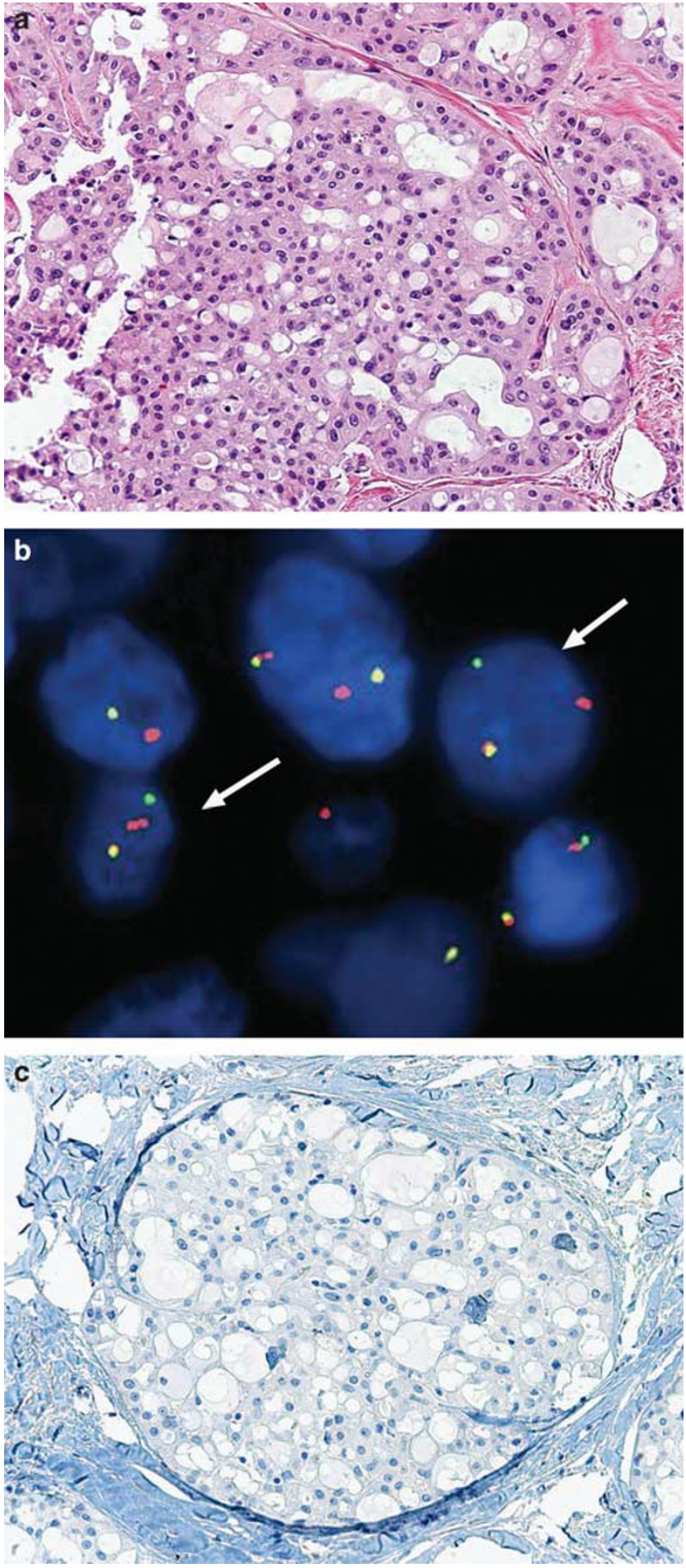

Figure 4 Mammary analog secretory carcinoma. (a) Tumor shows cribriform nests of cells with moderate amounts of somewhat vacuolated eosinophilic cytoplasm and luminal secretions reminiscent of non-specific ductal cells and vacuolated cells in acinic cell carcinoma (H\&E, $\times 200$ ). (b) ETV6 FISH break apart probe showing cells with one split orange signal indicative of a translocation (arrows). (c) Most mammary analog secretory carcinomas were DOG1 negative $(\times 200)$.

of distal intercalated ducts. Polymorphous lowgrade adenocarcinoma is one of a few tumors thought to recapitulate the phenotype of distal intercalated duct region based on immunohistochemical and ultrastructural findings, and one of the initial names for this entity was 'terminal duct carcinoma'. ${ }^{24,25}$ Other tumors that occasionally enter into the differential diagnosis such as mucoepidermoid carcinomas were only focally and weakly positive, mainly in mucocytes. Only one myoepithelial tumor showed any staining for DOG1, and this tumor did not have epithelioid, clear cell, or oncocytic morphology that would even raise the consideration for acinic cell carcinomas. Warthin tumors that rarely enter into the differential diagnosis were also uniformly negative. One caveat is that we did not test any 'cystadenocarcinomas, not otherwise specified,' which as a class of lesions can show overlap with papillary cystic variant of acinic cell carcinoma.

Perhaps, most interesting to the differential diagnosis of acinic cell carcinomas is the recently described entity, mammary analog secretory carcinomas. Historically, mammary analog secretory carcinomas were most often classified as 'zymogen granule poor' acinic cell carcinomas or as adenocarcinoma, not otherwise specified. ${ }^{14-16}$ These carcinomas are composed of cells with moderateto-abundant eosinophilic, vacuolated cytoplasm show a lobulated growth pattern with solid, papillary, microcystic, or glandular spaces filled with dense eosinophilic mucinous material. Tumors are characteristically strongly S100, vimentin, mammaglobin, and STAT5a positive ${ }^{14-16,26}$ No true serous acinar differentiation is noted in these tumors. Mammary analog secretory carcinomas also recapitulate the morphology of juvenile secretory carcinoma of the breast (hence the name, mammary analog secretory carcinoma) and harbor the same ETV6NRTK3 translocation. The lack of true serous acinar differentiation, the characteristic morphology, and a reproducible translocation justify separation of mammary analog secretory carcinomas from acinic cell carcinomas. And though we only had a few cases to test, the DOG1 staining profile in mammary analog secretory carcinomas was indeed different from acinic cell carcinomas, and more in keeping with an acinar-intercalated duct junction phenotype. Only one case showed staining reminiscent of acinic cell carcinomas. Thus, DOG1 adds yet another marker to the armament (including mucicarmine, S100, and mammaglobin) that is readily available to help distinguish mammary analog secretory carcinomas from acinic cell carcinomas.

In completing our survey of salivary gland tumors, we encountered a high frequency of DOG1 positivity in adenoid cystic carcinoma and epithelial-myoepithelial carcinoma, though not as consistently as seen in acinic cell carcinomas. Our findings with adenoid cystic carcinoma are somewhat similar to those of Lopes et $a l^{27}$ who found positivity in 3/4 tumors using the K9 antibody. Additionally, we found that more solid adenoid cystic carcinoma and adenoid cystic carcinoma with high-grade 

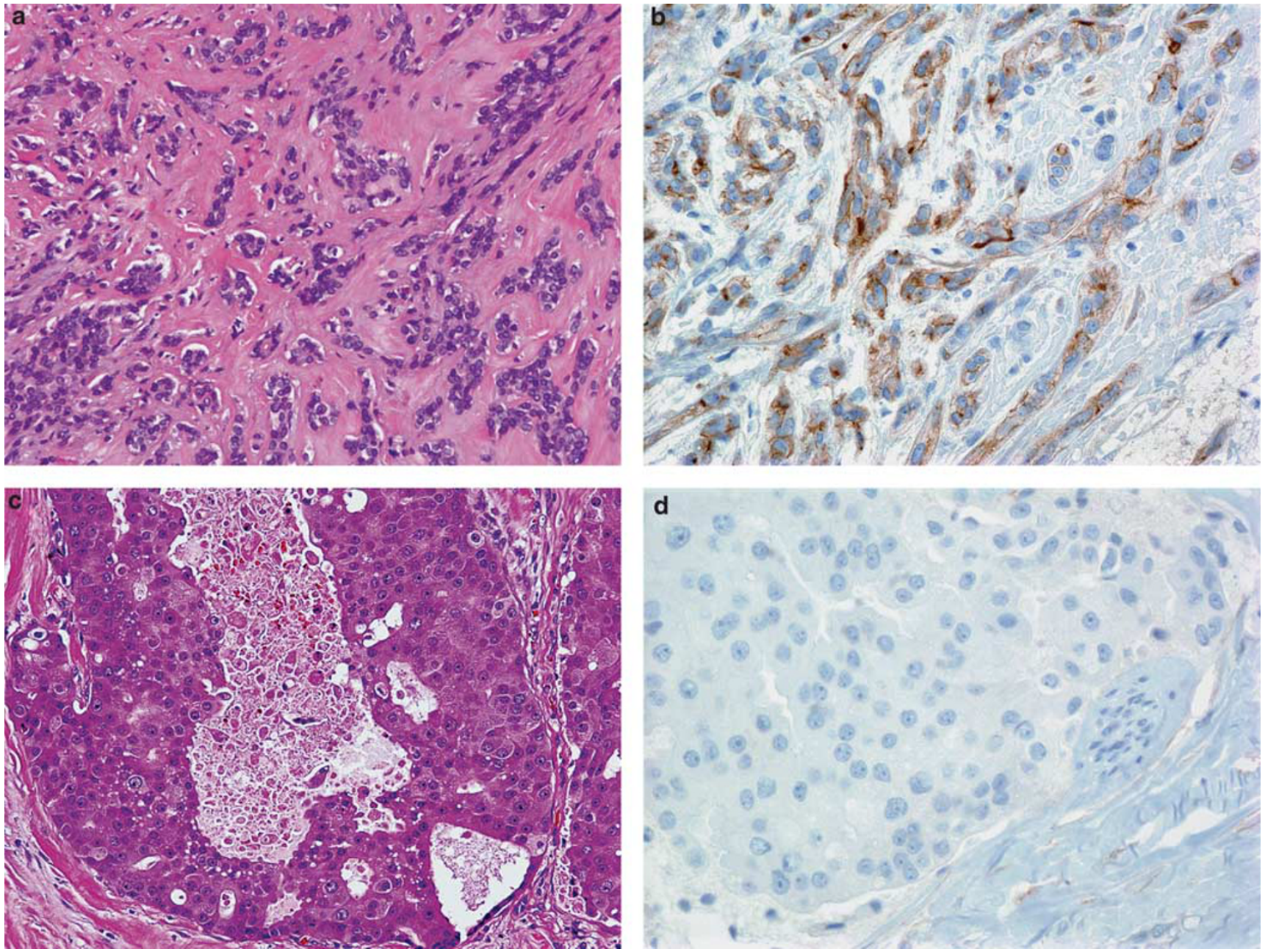

Figure 5 DOG1 staining in other ductal neoplasms. (a) Polymorphous low-grade adenocarcinomas (H\&E, $\times 200)$. (b) DOG1 was focally positive in a subset of tumors in an apical membranous pattern. This particular case was diffusely positive $(2+)$ with some complete membranous staining as well $(\times 400)$. (c) Salivary duct carcinoma $(H \& E, \times 200)$. (d) All tested cases were negative. Note the perineural invasion in this case (bottom right) $(\times 400)$.

transformation were more frequently DOG1 negative as compared with tubular and cribriform predominant adenoid cystic carcinoma. Adenoid cystic carcinoma and epithelial-myoepithelial carcinoma tumors fit into the 'biphasic' category of salivary gland tumors. These are tumors that comprising a bilayered arrangement of inner (luminal) ductal and outer (abluminal) basal and/or myoepithelial cells. This pattern is often readily visible by routine light microscopy, and by immunostaining shows a sharp distinction between luminal and abluminal components. As such, neither adenoid cystic carcinoma nor epithelial-myoepithelial carcinoma is usually in the differential diagnosis for acinic cell carcinomas (a 'monophasic' acinar malignancy) and do not invalidate the use of DOG1 as discussed above.

However, this is still of interest, particularly given the unique pattern of staining identified. The ductal constituents of these two tumor types, when positive, showed a heterogeneity and apical mem- branous pattern reminiscent of normal intercalated ducts. But surprisingly, the myoepithelial components were frequently positive and showed a complete membranous and even cytoplasmic pattern of DOG1 reactivity in adenoid cystic carcinoma and epithelial-myoepithelial carcinoma. This contrasts with normal salivary gland myoepithelial and basal cells, which were uniformly negative suggesting that this is a 'transformed' phenotype in salivary tumors. It must be noted, however, that this may not hold true at other sites with myoepithelial cells. For instance, this pattern of staining has been described in normal basal/myoepithelial cells in breast and prostate. $^{2,27}$ Any role for an anion channel such as DOG1 in myoepithelial cells, normal or neoplastic at any site is currently unknown.

Another interesting finding was that this dual staining pattern was mainly restricted, in our study, to biphasic malignant tumors and a few putative precursor lesions. One case of intercalated duct 

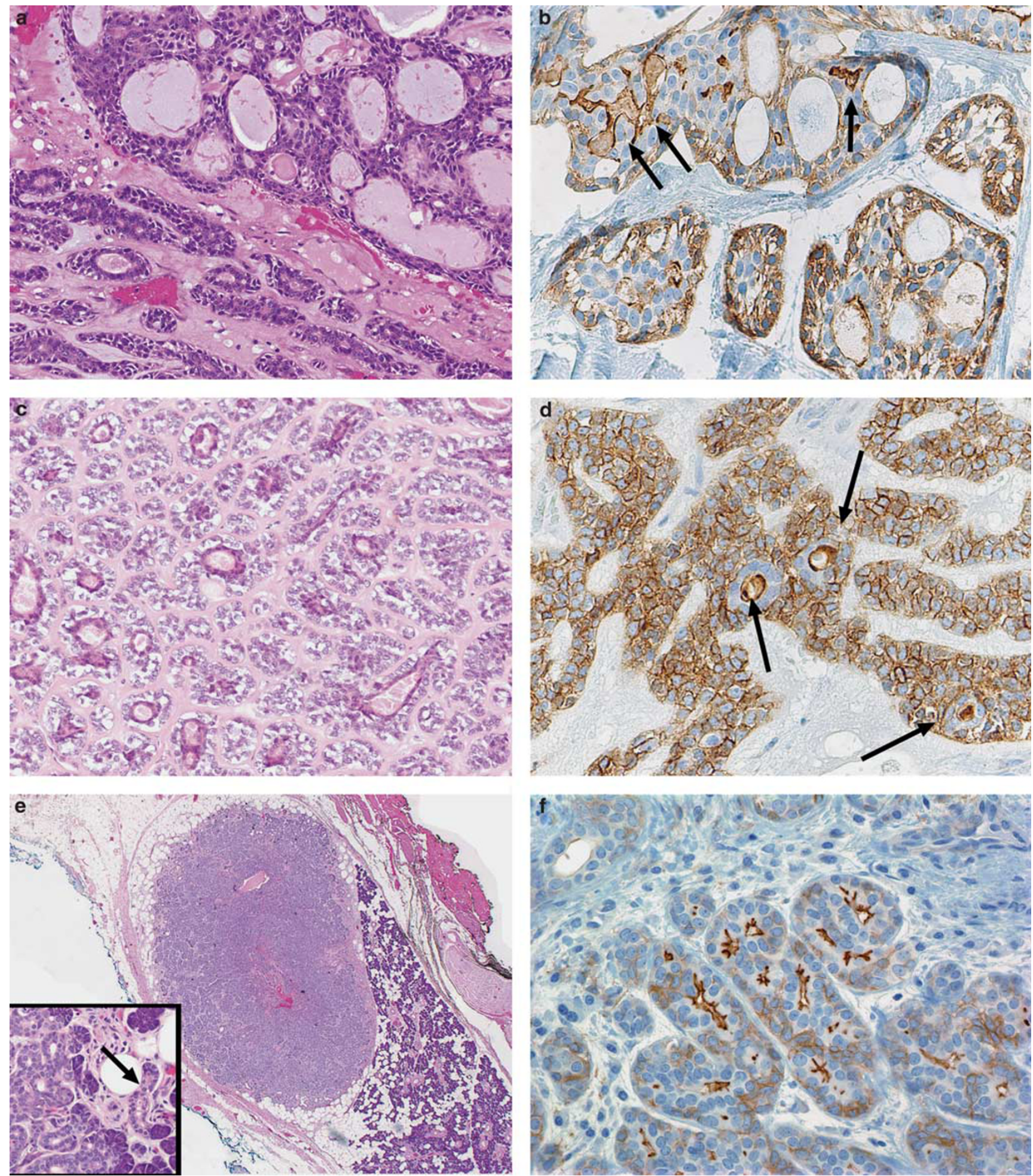

Figure 6 DOG1 staining in adenoid cystic carcinomas, epithelial-myoepithelial carcinomas, and intercalated duct lesions. (a) Adenoid cystic carcinoma, cribriform pattern $(\mathrm{H} \& \mathrm{E}, \times 200)$. (b) DOG1 frequently showed an apical membranous staining pattern $(2+$ in this case) in the ductal component (arrows), and a cytoplasmic pattern in the abluminal myoepithelial cells $(1+$ in this case) $(\times 400)$. (c) Epithelial - myoepithelial carcinoma with a classic clear cell myoepithelial morphology (H\&E, $\times 200)$. (d) Similarly to adenoid cystic carcinomas, epithelial - myoepithelial carcinoma had moderate apical-luminal DOG1 staining $(2+$ arrows) complete membranous staining of the abluminal myoepithelial cells $(2+)(\times 400)$. (e) A 3-mm intercalated duct adenoma with partial encapsulation (H\&E, $\times 20)$. Inset shows transition of adenoma from adjacent acini. The proliferation consists of ducts the same size and caliber of a normal intercalated duct (arrow). (f) DOG1 shows staining of ductal and myoepithelial components reminiscent of epithelial-myoepithelial carcinoma $(\times 400)$. 
hyperplasia in association with an epithelial-myoepithelial carcinoma, and one intercalated duct adenoma showed a similar staining pattern to epithelial-myoepithelial carcinoma and adenoid cystic carcinoma. Intercalated duct lesions are not well characterized but have been mainly seen in association with epithelial-myoepithelial carcinoma and basal cell salivary gland neoplasms (adenoma and carcinoma). Thus, if these lesions can truly serve as precursors to epithelial-myoepithelial carcinoma, then it is reasonable to find that some of them may show a similar 'transformed' DOG1 staining pattern in the myoepithelial component. ${ }^{28,29}$ On the other hand, the main biphasic benign tumor category tested was pleomorphic adenoma, which was also largely negative. One limitation to this study however is that we did not test any basal cell adenomas and only tested one basal cell adenocarcinoma (focally positive), which are also often considered as biphasic tumors. Additionally, tumors composed only of myoepithelial cells were negative in all but one case of myoepithelial carcinoma that showed only focal staining.

Thus in summary, we confirm high levels of ANO1/DOG1 expression in human salivary tissues with predominant apical membranous serous acinar staining pattern. Acinic cell carcinomas almost uniformly show diffuse and intense DOG1 immunopositivity, a finding that can be used in conjunction with the traditional periodic acid-Schiff reaction after diastase treatment to distinguish acinic cell carcinomas from other morphologic mimics, particularly mammary analog secretory carcinomas. A subset of biphasic malignancies, adenoid cystic carcinoma, and epithelial-myoepithelial carcinoma show a distinctive DOG1 staining profile in both ductal and myoepithelial cell components.

\section{Disclosure/conflict of interest}

The authors declare no conflict of interest.

\section{References}

1 West RB, Corless CL, Chen X, et al. The novel marker, DOG1, is expressed ubiquitously in gastrointestinal stromal tumors irrespective of KIT or PDGFRA mutation status. Am J Pathol 2004;165:107-113.

2 Lee CH, Liang CW, Espinosa I. The utility of discovered on gastrointestinal stromal tumor 1 (DOG1) antibody in surgical pathology-the GIST of it. Adv Anat Pathol 2010;17:222-232.

3 Miettinen M, Wang ZF, Lasota J. DOG1 antibody in the differential diagnosis of gastrointestinal stromal tumors: a study of 1840 cases. Am J Surg Pathol 2009;33: 1401-1408.

4 Carles A, Millon R, Cromer A, et al. Head and neck squamous cell carcinoma transcriptome analysis by comprehensive validated differential display. Oncogene 2006;25:1821-1831.
5 Caputo A, Caci E, Ferrera L, et al. TMEM16A, a membrane protein associated with calcium-dependent chloride channel activity. Science 2008;322:590-594.

6 Schroeder BC, Cheng T, Jan YN, et al. Expression cloning of TMEM16A as a calcium-activated chloride channel subunit. Cell 2008;134:1019-1029.

7 Yang YD, Cho H, Koo JY, et al. TMEM16A confers receptor-activated calcium-dependent chloride conductance. Nature 2008;455:1210-1215.

8 Ferrera L, Caputo A, Galietta LJ. TMEM16A protein: a new identity for $\mathrm{Ca}(2+)$-dependent $\mathrm{Cl}$ channels. Physiology (Bethesda) 2010;25:357-363.

9 Hwang DG, Qian X, Hornick JL. DOG1 antibody is a highly sensitive and specific marker for gastrointestinal stromal tumors in cytology cell blocks. Am J Clin Pathol 2011;135:448-453.

10 Almaca J, Tian Y, Aldehni F, et al. TMEM16 proteins produce volume-regulated chloride currents that are reduced in mice lacking TMEM16A. J Biol Chem 2009;284:28571-28578.

11 Kunzelmann K, Kongsuphol P, Aldehni F, et al. Bestrophin and TMEM16-Ca(2+) activated $\mathrm{Cl}(-)$ channels with different functions. Cell Calcium 2009;46:233-241.

12 Ousingsawat J, Martins JR, Schreiber R, et al. Loss of TMEM16A causes a defect in epithelial Ca2+-dependent chloride transport. J Biol Chem 2009;284: 28698-28703.

13 Ferrera L, Caputo A, Ubby I, et al. Regulation of TMEM16A chloride channel properties by alternative splicing. J Biol Chem 2009;284:33360-33368.

14 Skalova A, Vanecek T, Sima R, et al. Mammary analogue secretory carcinoma of salivary glands, containing the ETV6-NTRK3 fusion gene: a hitherto undescribed salivary gland tumor entity. Am J Surg Pathol 2011;34:599-608.

15 Chiosea SI, Griffith C, Assaad A, et al. The profile of acinic cell carcinoma after recognition of mammary analog secretory carcinoma. Am J Surg Pathol 2012; 36:343-350.

16 Chiosea SI, Griffith C, Assaad A, et al. Clinicopathologic characterization of mammary analogue secretory carcinoma of salivary glands. Histopathology 2012; e-pub ahead of print.

17 Bergmann F, Andrulis M, Hartwig W, et al. Discovered on gastrointestinal stromal tumor 1 (DOG1) is expressed in pancreatic centroacinar cells and in solid-pseudopapillary neoplasms-novel evidence for a histogenetic relationship. Hum Pathol 2011;42: 817-823.

18 Ardeleanu C, Arsene D, Hinescu M, et al. Pancreatic expression of DOG1: a novel gastrointestinal stromal tumor (GIST) biomarker. Appl Immunohistochem Mol Morphol 2009;17:413-418.

19 Davis AJ, Forrest AS, Jepps TA, et al. Expression profile and protein translation of TMEM16A in murine smooth muscle. Am J Physiol Cell Physiol 2010; 299:C948-C959.

20 Gomez-Pinilla PJ, Gibbons SJ, Bardsley MR, et al. Ano1 is a selective marker of interstitial cells of Cajal in the human and mouse gastrointestinal tract. Am J Physiol Gastrointest Liver Physiol 2009;296:G1370-G1381.

21 Huang X, Godfrey TE, Gooding WE, et al. Comprehensive genome and transcriptome analysis of the $11 q 13$ amplicon in human oral cancer and synteny to the 7F5 amplicon in murine oral carcinoma. Genes Chromosomes Cancer 2006;45:1058-1069. 
22 Childers EL, Ellis GL, Auclair PL. An immunohistochemical analysis of anti-amylase antibody reactivity in acinic cell adenocarcinoma. Oral Surg Oral Med Oral Pathol Oral Radiol Endod 1996;81:691-694.

23 Ihrler S, Blasenbreu-Vogt S, Sendelhofert A, et al. Differential diagnosis of salivary acinic cell carcinoma and adenocarcinoma (NOS). A comparison of (immuno-)histochemical markers. Pathol Res Pract 2002;198:777-783.

24 Batsakis JG, Pinkston GR, Luna MA, et al. Adenocarcinomas of the oral cavity: a clinicopathologic study of terminal duct carcinomas. J Laryngol Otol 1983;97:825-835.

25 Araujo V, Sousa S, Jaeger M, et al. Characterization of the cellular component of polymorphous low-grade adenocarcinoma by immunohistochemistry and electron microscopy. Oral Oncol 1999;35:164-172.
26 Griffith C, Seethala R, Chiosea SI. Mammary analogue secretory carcinoma: a new twist to the diagnostic dilemma of zymogen granule poor acinic cell carcinoma. Virchows Arch 2011;459:117-118.

27 Lopes LF, West RB, Bacchi LM, et al. DOG1 for the diagnosis of gastrointestinal stromal tumor (GIST): comparison between 2 different antibodies. Appl Immunohistochem Mol Morphol 2010;18:333-337.

28 Chetty R. Intercalated duct hyperplasia: possible relationship to epithelial-myoepithelial carcinoma and hybrid tumours of salivary gland. Histopathology 2000;37:260-263.

29 Weinreb I, Seethala RR, Hunt JL, et al. Intercalated duct lesions of salivary gland: a morphologic spectrum from hyperplasia to adenoma. Am J Surg Pathol 2009; 33:1322-1329. 